nellement opposé aux excès d'un étatisme stérilisant; nous comptons sur vons pour défendre les intérêts de l'industrie nationale en contribuant de toute votre influence à lui conserver la liberté d'action dont elle a besoin.

Enfin, Monsieur le Ministre, permettez-nous d'appeler votre bienvellante attention sur la patente dont sont frappés les usines et ateliers qui empruntent au dehors, sous forme de courant électrique, l'énergie motrice nécessaire à leur fonctionnement.

Vous savez que l'Administration des Contributions Directes a cru devoir, au cours de ces dernières années, faire entrer clans l'estimation de la valeur locative de ces usines et ateliers, pour l'établissement du droit proportionnel des patentes, non seulement la valeur des machines installées pour transformer en travail mécanique l'énergie électrique qui leur est amenée du dehors, mais, encore, la valeur marchande de cette énergie ; cependant, le courant électrique est un produit qui peut être absolument assimilé à la houille employée pour le chauffage des machines à vapeur, et jamais les Contributions Directes n'avaient songé à ajouter, à la valeur locative des machınes à vapeur d'une usine, la valeur de la houille achetée par l'usinier pour mettre ces machines en mouvement.

A la suite de très vives réclamations de la plupart des Chambres de Commerce de France, une proposition de loi - signée d'un très grand nombre de délputés - a été présenlée d̀ la Chambre par M. le député Cazeneuve, pour régler celte grave question dans le sens qu'exigent, on peut le dire, non seulement l'idée fiscale, mais encore le simple bon sens. et nous sommes heureux, Monsieur le Ministre, de rappeler que votre nom figure parmi ceux des signataires de celte proposition de loi.

L'Administration des Contributions Directes a reconnu olle-meme qu'elle ne pourrait pas lutter contre un tel mouvement d'opinion publıque, et $M$. le Ministre des Finances vient de déproser, il y a peu de temps, sur le Bureau de la Chambre un projet de loi dont le premier paragraphe reproduit dans des termes un peu différents la proposition Cazenouve, mais le second paragraphe comporte des restrictions dont nous avons le devoir de vous souligner le danger.

D'après ce second paragraphe, la valeur locatıve, qui servirait cle base au droit proportionnel de la patente des usines ch ateliers recevant leur force motrire du dehors, serait élablıe par comparaison avec la valeur locative des autres ctablissements industriels de la même région.

Fin d'autres termes, un modeste atelter de serrurerie, qui falt mouvoir ses tours et ses diverses machines-outils au moyen d'e courant électrigne emprunté à la distrubution de la ville, sera taxé comme s'll avait installé dans son atelier une coûteuse machine à vapeur accompissant le même travail.

Ne vo'yez-vous pas, Monsieur le Ministre, qu'une telle fiscalito, qui ne tient aucun compte clu progrès de la Science applicquée, va à l'encontre des intêrêts les plus évilents du pays?

Il y a un intérêt social de premier ordre à ce que le Gouvernement, loin de décourager amsı le progrès, favorise autant qu'il le pourra la transformalion du vieil outlllage mćcanique et son remplacement par l'outillage perfectionné que l'énergie électrique, distribuée sur tout le territoire, actionnera dans les conditions les plus économiques, et au grand avantage de la santé physique et morale de nos populatıons ouvrières.

Les réseaux de distribution peuvent, en effet, permettre de décongestionner les villes, de disséminer l'industrie, de faire renaître dans bien des cas les ateliers de famille. Liélectricité est linstrument le plus souple et le plus puissant que nous ayons à notre disposition pour réaliser l'idéal de la Soience appliquée.
Cet idéal, c'est de dominér les forces de la nature, de les asservir, de les domestiquer, d'en faire les servantes dociles de l'homme, de sorte que l'ouvrier, au lieu d'user péniblement sa force musculaire, ne soit plus jamais que le conducteur intelligent d'une machine produsant sans fatigue les ouvrages les plus compliqués.

N'est-ce pas aller à lencontre des intérêts les plus évidents. du pays que de ralentir par des mesures fiscales l'essor de cette transformation de l'outillage national ?

Nous vous prions instamment, Monsieur le Ministre, d'appeler l'attention du Gouvernemnet sur les inconvénients que présenterait l'adoption d'un tel projet de loi et pour lus demander, au contraire, de ne pas s'opposer à l'adoption de' la proposition de M. Cazeneuve et de ses collègues.

Depuis le peu de temps que vous occupez le Ministère dı Commerce, nous avons pu apprécier votre esprit libéral; volre fonction est d'être le protecteur de IIndustrie; nous. rometions entre vos mains notre défense, persuadés que vous penserez avec nous que les intérêts dont nous nous préoccupons rie sont pas seulemient les intérêts privéls de quelques industriels, mais quils concordent avec l'intérêt général de la nation.

\section{INSTALLATION HYDRO-ÉLECTRIQUE DE LA PESCARA}

La Società Italiand Electrochimica a obtenu la concession d'une dérivation de $30 \mathrm{~m}^{3}$ à la seconde de la rivière Pescara, sur la commune de Popoli, province d'Aquila, depuis le confluent du Tirino jusqu'à Piano d'Orte, lui permettant de réaliser une chute totale de $99 \mathrm{~m} 20$. Pour diverses raisons, à la fois techniques et économiques, la Société a divisé la chute on deux tronçons. Le premier utilise unø chute de $2 \bar{i}^{\mathrm{m}} 60$, a vec une usine hydro-électrique à «Tre Monti ${ }_{\text {, }}$ capable de développer 8300 chevaux; le second utilise une chute de $71 \mathrm{~m} 60$, avec usine hydro-électrique à Piano d'Orte, d'une puissance de 21500 chevaux.

Nous allons donner ici quelques renseignements sur l'aménagement de la première chute.

Le barrage de dérivation a $30^{\mathrm{m}} 85$ de largeur, et est constitué par 5 vannes mobiles verticales, de $5 \mathrm{~m}$. de largen" et de $2 \mathrm{~m}$. de hauteur, qui s'appuient sur des piles intermé. diaires de 1 m 30 de largeur.

La prise d'eau se fait au moyen de 12 ouvertures obliques, de $3 \mathrm{~m}$. de largeur sur $0^{\mathrm{m}} 80$ de hauteur, séparées par des piles en béton à ossature métallique. Le seuil de ces ouvertures est à $0^{\mathrm{m}} 90$ en contrehaut de celui des vannes du barrage, de manière à éviter l'introduction des galets dans lachambre de décentation, ainsi que dans le canal d'amenée, Chaque ouverture est ell outre munie d'une vanno d'arrêt.

Le bassin de décantation fait suite à la prise d'eau, et est muni de deux vannes de purge accolées, de $7^{\mathrm{m}} 60$ de largeur totale. Cette chambre est divisée en deux parties par une grille, de $34 \mathrm{~m}$. de longueur. Le niveau de l'eall y est maintenu conslant au moyen d un déversoir de superficie, qui rejette automatiquement dans la Pescara les eaux surabondantes. En tête du canal de dérivation se trouvent 2 vannes de $3^{\mathrm{m}} 40 \times 2^{\mathrm{m}} 95$, destinées à l'isoler en cas d'accident.

Le canal d'amenée a d'abord une section rectangulaire, de $7^{\text {in}} 60$ de largeur, sur les 23 premiers mètres, puis il prend ensuite une section trapézoïdale avec parois maçonnées, inclinées à $45^{\circ}$. Le canal a une longueur de $314 \mathrm{~m}$. et une pente de 0,5 millimètre par mètre; sa section transversale utile est de $25,80 \mathrm{n}^{2}$, el sa capacité de $40 \mathrm{~m}^{3}, \dot{a}$ la seconde. A la suite de co canal à ciel ouvert se trouve une galerie maçonnée de $2235 \mathrm{~m}$. de longueur. La section transversale de cette galerie est composée d'un rectangle, de $3^{m} 25$ de hauteur sur $4^{m} 50$ de lar'geur, surmonté d'une voùto de $0^{\text {in }} 90$ de flèchie; la pento est de 0,8 millimètre par mètre, ot la surface utile de 17,40 mètres carrés. 
La chambre de mise en chargeà une capacité de $1750 \mathrm{~m}^{3}$, et est entièremont creusée dans le rocher. Elle est constiluée par une galerie principale, de $30 \mathrm{~m}$. de longueur et de $8 \mathrm{~m}$. de largeur, d'où se détachent trois autres galeries, à angle droit avec la première, de $13 \mathrm{~m}$. de longueur et ayant péspectivement $7 \mathrm{~m}, 7 \mathrm{~m}$. et $3 \mathrm{~m}$. de largeur. A l'extrémité des deux premières galeries s'en détachent deux autres, de $7 \mathrm{~m}$. de longueur et de $5 \mathrm{~m} 80$ de largeur, d'où partent les 4 conduites forcées principales. Ultérieurement, une cinquième conduite partira de la troisième galerie précitée. La cliambre de mise en charge est munie d'un déversoir, de vannes de purge, de grilles et de vannes d'arrêt placies irnmédiatement en avanl des conduites forcées.

Les 4 conduites forcées principales ont 2 "20 de diamètre et $100 \mathrm{~m}$. de longueur; une petite conduite de $0 \mathrm{~m} 60$ alimente en outre lesexcitatrices. Elles ont une épaisseur de 5 à $7 \mathrm{~mm}$., et sont renforcées tous les $1^{\mathrm{m}} 80$ par des anneaux en fer cornic̀re.

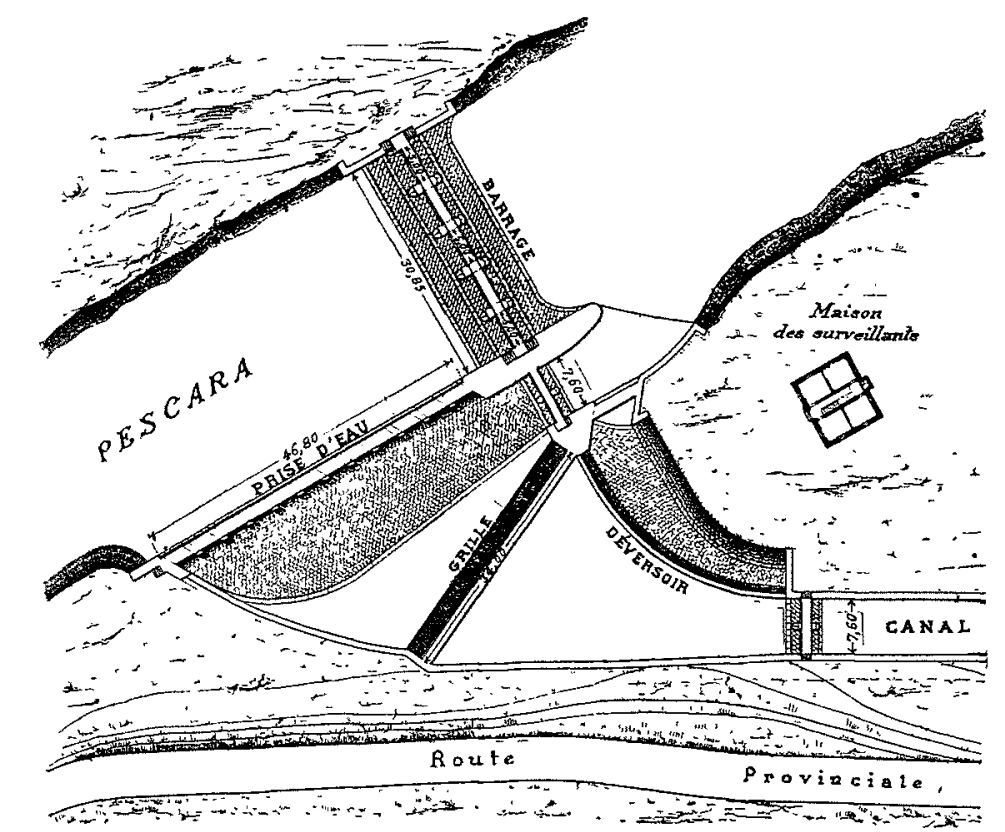

L'usine génératrice de Tre Monti comprend un corps principal de bàtiment, de $45 \mathrm{~m}$. de lons sur $15 \mathrm{~m}$. de large et $12 \mathrm{~m}$. de haut, et dune annexe à deux étages, de $34 \mathrm{~m} 50$ sur $7 \mathrm{~m}$. dans lequel se trouvent, aurez-de-chaussée, lo tableau général et les transformateur's, et, au-dessus, le départ de la ligne de transmission.

l'usine comporte à l'heure actuelle 4 groupes électro gines de $2800 \mathrm{HP}$, dont un de réserve et deux group $\mathrm{c}$ d'excitation de $150 \mathrm{HP}$ chacun. Les grosses turbines sont doubles, du type Francis, centripète parallèle, à aspiration les aubes sont mobiles. Les alternateurs sont à induit fixe el inducteurs tournants. Ils produisent du courant triphasé ả 6000 volts, 45 périodes, à la vitesse de 270 tours par minute. Les exitatrices tournent à 400 tours.

Une partie du courant alternatif esl élevé à la tension de 25000 volts au moyen de 4 transformaleurs monophasés (lont 1 de réserve) de $150 \mathrm{~K} . \mathrm{V}$ A. connectés en étoile. Le r'cste est utilisé directement à la tension de 600 volts.

De la centrale de Tro Monti partent 3 lignes de distribution, qui sont munies chacune d'interrupteurs automatiques el de déchargeurs Siemens Wurtz à jet d'eau.

Une première ligne, longue de $3,2 \mathrm{kms}$, fournit du courant à 600 volts à la Socielà Italiana dell' Aluminio à Bussi. Elle est constitúée par 6 fils de $78 \mathrm{~mm}^{2}$ de section, supportés pal des poteaux métalliques.

Une seconde ligne, de $10 \mathrm{kms}$, fournit du courant à $600 i$ volts à la So:iela dei prodoti azotati, à Piano d'Orte. Elle est constituée par 3 fils de $40 \mathrm{~mm}^{2}$, supportés par des poteaux en bois.
Enfin, une troisième ligne fournitdu courant à 25000 volts à la Società Imprese Elettriche Abruzsesi, à Castellamare ct à Francavilla. Elle est constituée par 3 fils de $5 \mathrm{~mm}$. de diamètre.

M. P.

\section{THEORIE DU DEVERSOIR}

Notes de M. Boussinesa, de l'Académie des Sciences (Suile)

Théorie de l'écoulement sur un déversoir vertical en mince paroi et sans contraction latérale : cas de la nappe ondulée, et son raccordement au cas de la nappe plongeante $\left(^{*}\right)$.

I. - Lorsque, dans l'écoulement de l'eau sur un déversoir vertical en mince paroi, tenant toute la largeur du canal compris entre deux murs verticaux parallèles, la nappc de déversement est noyée en dessous par une masse d'eau tourbillonnante, dont la pression au niveau du seuil égale une fraction donnée $N^{\prime}$ de la pression pgh quis'y exercerait, à l'état de repos, si le niveau avait partout, au-dessus du seuil, sa hauteur $h$ d'amont (dite hauteur de charge), le coefficient $m$ du débit $m h / \overline{2 g h}$ par unité de longueur du déversoir est une certane fonction de $N^{\prime}$, dont j'ai indiqué ou même effectué à très peu près le calcul dans une note du I er juillet 1907, pour les valcurs de $N^{\prime}$ comprises entre - $\infty$ et 0,8 . A cette limite $N^{\prime}=0,8$, un certain parametre $k$, relié comme l'indique la formule:

$$
\frac{R_{0}}{r_{1}}=\frac{k}{1-k}
$$

au quotient du rayon $R_{0}$ de courbure des filets fluides inférieurs (à la traversée de la section contractée) par l'épais-

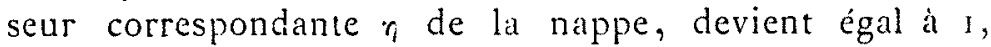
après avoir crû à partir de zéro pendant que $N^{\prime}$ passait de - $\infty$ à 0,8 . Donc, à ce moment où $N^{\prime}=0,8$, les filets fluides sont sensiblement rectilignes à la traversée de la section contractée, après y àvoir été, au début, fortement concaves vers le bas.

Or, les accroissements successifs de $N^{\prime}$ se produiscnt, effectivement, à mesure qu'on relève le niveau d'ayal ou niveau de l'eau dans le canal de fuite, en abaissant de plus en plus une vanne située à quelque distance en aval du déversoir; et ce niveau excède notablement le seuil au moment oû $N^{\prime \prime}=0,8$. Dès lors, la nappe, sans c.surbure sensible à la traversée de la section contractée (où elle est presque horizontale encore), n'a plus à descendre pour se joindre à l'eau stagnante ou tourbillonnante d'aval; ct elle cesse de plonger au sein de cette eau, ou de s'y noyer complètement, pour s'étaler simplement à sa surface et ne rester dès lors nojéc qu'en dessons. M. Bazin a observé, cn eflet, qu'elle se tient à la surface libre; et il l'a appelée nappe ondulée, en raison de quelques ondulations qu elle y présente.

Il est clair que, si l'eau d'aval se relève encore plus, et rend supérieure à 0,8 la pression relative $N$ ' sous la nappe,

(*) Séance du 23 mars 1908. 